\title{
NECESIDAD DE TRATAMIENTO BUCAL Y CALIDAD DE VIDA EN EL ADULTO MAYOR
}

\author{
${ }^{1}$ Gloria Cristina Aránzazu Moya, ${ }^{2}$ Anne Alejandra Hernández Castañeda, ${ }^{3}$ Maira Alejandra Gutiérrez Sánchez, ${ }^{3}$ Daniel Felipe Agudelo Prada \\ ${ }^{1}$ Odontóloga U. Santo Tomas, Especialista en Patología y Medios Diagnósticos U. El Bosque, Docente U. Santo Tomás, Colombia. ${ }^{2}$ Odontóloga U. Santo Tomás, Joven investigador \\ Colciencias - U. Santo Tomás, Colombia. ${ }^{3}$ Odontólogo U. Santo Tomás, Colombia.
}

Autor responsable de correspondencia: Gloria Cristina Aranzazu Moya

Correo electrónico: gloriacristina33@mail.ustabuca.edu.co

\section{RESUMEN}

Objetivo: establecer las necesidades de tratamiento bucal y su influencia en la calidad de vida relacionada con la salud bucal en el adulto mayor.

Materiales y métodos: se realizó un estudio de corte transversal en adultos mayores institucionalizados de seis hogares geriátricos del municipio de Floridablanca, Santander (Colombia). Se establecieron las necesidades de tratamiento según la Organización Mundial de la Salud (OMS) y la calidad de vida relacionada con la salud bucal según la versión en español del Geriatric Oral Health Assesment Index (GOHAI). Se obtuvieron medidas de tendencia central y dispersión para las variables cuantitativas y proporciones para las cualitativas. También, se realizó un test de $\mathrm{Chi}^{2}$ o Exacto de Fisher según correspondiera. Un valor de $\mathrm{p} \leq 0,05$ fue considerado significativo.

Resultados: de los 111 adultos mayores examinados, el 62,2\% necesitaba tratamiento especial por su enfermedad sistémica. El 13,5\%, requería intervención de tejidos con manejo clínico mientras que el $37,8 \%$ de la población necesitaba un tratamiento especial por flujo salival. El 44,4\%, requería raspaje y alisado radicular a campo cerrado y un manejo preventivo con educación en higiene bucal en el $86,5 \%$. Las frecuencias de auto percepción y calidad de vida no mostraron una asociación estadísticamente significativa.

Conclusiones: las tres principales necesidades de tratamiento en su orden fueron: educación en higiene bucal, tratamiento especial por enfermedad sistémica y tratamiento periodontal. Las necesidades de tratamiento fueron más importantes en los pacientes con bajos puntajes de calidad de vida según el GOHAI, pero no se observó una diferencia estadísticamente significativa. [Aranzazu GC, Hernández AA, Gutiérrez MA, Agudelo DF. Necesidad de tratamiento bucal y calidad de vida en el adulto mayor. Ustasalud 2013; 12: 47 - 54]

Palabras clave: Anciano, Calidad de vida, Salud bucal.

\section{ORAL TREATMENT NEEDS AND OLDER PEOPLE'S QUALITY OF LIFE}

\begin{abstract}
Objective: to assess the oral treatment needs and its effect on oral health related quality of life in older adults.

Methods: a cross sectional study in six elderly nursing home care from the city of Floridablanca, Santander (Colombia) was done. Treatment needs were established according to the World Health Organization (WHO) and the oral health related quality of life according to the Spanish version of the Geriatric Oral Health Assessment Index (GOHAI). Measures of central tendency and dispersion for quantitative variables and proportions for qualitative ones were obtained. Chi ${ }^{2}$ test or Fisher's Exact was used as appropriate. A value of $\mathrm{p} \leq 0.05$ was considered significant.

Results: of the 111 seniors surveyed, $62.2 \%$ needed special treatment for systemic diseases. 13.5\% required intervention by clinical management while $37.8 \%$ of the population needed special treatment for salivary flow. $44.4 \%$ required deep scaling and root planning and oral hygiene education was needed in $86.5 \%$. The frequencies of self-perception and oral health related quality of life did not show any statistically significant association.

Conclusions: the three main treatment needs in their order were oral hygiene education, special treatment for systemic disease and periodontal treatment. Treatment needs were more important in patients with low quality of life scores according GOHAI but no statistically significant difference was observed.
\end{abstract}

Key words: Aged, Quality of life, Oral health

Recibido para publicación: diciembre 10 de 2012. Aceptado para publicación: marzo 13 de 2013. 


\section{INTRODUCCIÓN}

La población mundial muestra un crecimiento del grupo poblacional del adulto mayor posiblemente, debido a factores como la natalidad, mortalidad, migración y otros que han modificado a la sociedad moderna. ${ }^{1,2}$ Dicho incremento ha generado cierta vulneración en la población adulta mayor debido a que se ha descompensado la balanza de atención a los padecimientos de salud, que podrían llegar a afectar la calidad de vida del anciano. ${ }^{3}$ La Organización Mundial de la Salud (OMS) define al adulto mayor como "toda persona de 65 o más años, asumiendo que esta población se encuentra en la culminación de un proceso natural con deficiencias funcionales como resultado de cambios biológicos, psicológicos y sociales, condicionado por un fondo genético, los estilos de vida adoptados en la niñez, la acumulación de excesos en la juventud y los aspectos ambientales en que vive el individuo". $4,5 \mathrm{~A}$ medida que la edad aumenta la calidad de vida va a depender de la capacidad que la persona tenga de ser autónoma e independiente y de que pueda seguir los constantes cambios del medio actual desarrollándose acorde con sus preferencias y gustos personales, sin atenerse a nuevos modelos que lo condicionen a formas de vida que no sean de su elección.

Por su parte, la OMS define la calidad de vida como "la percepción individual de la propia posición en la vida dentro del contexto del sistema cultural y de valores en que se vive y en relación con sus objetivos, esperanzas, normas y preocupaciones", 6,7 también incluye en este concepto aspectos físicos, psicológicos, ambientales y sociales, ya que la calidad de vida propiamente dicha es un proceso de percepción personal de bienestar que se alcanza entre las condiciones de vida que se tienen y el grado de satisfacción con la misma. ${ }^{6,8,9}$

La calidad de vida del adulto mayor institucionalizado puede verse afectada si no cuentan con el apoyo familiar y son recluidos en centros de aslio que no cuenten con los recursos suficientes para cubrir las necesidades básicas en salud, alimentación y recreación, entre otros. Este aspecto se convierte en un factor coadyuvante para el deterioro de la salud tanto física como mental del anciano. ${ }^{6,10}$

Por otra parte, gran parte de la población adulta mayor toma medicamentos que tienen efectos secundarios en la cavidad bucal y desarrollan diversas alteraciones con mayor facilidad; ;1,12 además, el adulto mayor omite o refiere mal sus síntomas ya sea por miedo a la hospitalización, atribución de síntomas al envejecimiento o a factores sociales y culturales. ${ }^{13}$
Algunos adultos mayores muestran interés ante medidas para el mantenimiento de la salud, por tal razón, están dispuestos a invertir su dinero en tratamientos restauradores o en prevención. ${ }^{12,14} \mathrm{Sin}$ embargo, existe un creciente aumento de ancianos de bajos recursos y han sido caracterizados en el ámbito social como un grupo que en su gran mayoría se encuentra en condiciones de dependencia económica ya sea por parte de sus familiares o por donaciones externas o ajenas; ${ }^{12,15}$ este hecho debe considerarse al momento de indicar tratamientos dentales, pues es posible que éstos se limiten. Con respecto a los elementos de higiene bucal, sin ser extremadamente caros, suelen ser a menudo considerados artículos menos prioritarios por muchos pacientes, en relación con otros productos de carácter indispensable por lo que es conveniente adecuarse al presupuesto del paciente con los artículos de higiene bucal a indicar. ${ }^{14}$

La calidad de vida de toda persona está directamente relacionada con el buen funcionamiento de su cuerpo. ${ }^{16}$ En este sentido, la cavidad bucal es muy importante dado que por allí es precisamente por donde el alimento ingresa al organismo. Para el adulto mayor un mal funcionamiento de su cavidad bucal va a influir notablemente en su capacidad para alimentarse y poder comunicar sus dolencias. Desde el punto de vista estético puede generar vergüenza por su aspecto físico, a la par que se generaran otros problemas en el contexto social, cultural y en ciertas ocasiones religioso. ${ }^{17,18}$

Existe una gran variedad de patologías que se pueden encontrar en la cavidad bucal, entre estas la caries dental y la enfermedad periodontal; ${ }^{19,20}$ es muy importante comprender todos los factores, que hacen del individuo un objeto vulnerable, con frecuencia estos riesgos pueden dificultar una terapéutica preventiva ideal y emperorar el diagnóstico o prolongar su duración, por lo que se debe tener en cuenta que el proceso de envejecimiento produce en la cavidad bucal una serie de cambios graduales, irreversibles y acumulativos. ${ }^{14} \mathrm{Si}$ bien estos cambios varian entre los individuos pueden o no causar alteraciones en la función como pueden ser: la resequedad por la disminución salival debida a la edad o el consumo de medicamentos, desgaste de la superficie dental, perdida del hueso alveolar, disgueusia, menos capacidad defensiva y reparadora de tejidos, entre otras. ${ }^{19}$ Estas alteraciones provocan una mayor vulnerabilidad a los agentes traumáticos e infecciosos. ${ }^{12,14}$

A su vez, es importante evidenciar las demás enfermedades sistémicas que pueden estar presentes en el adulto mayor. Según informa el Departamento 
Administrativo Nacional de Estadística (DANE) desde 1995, las enfermedades del aparato circulatorio ocupan la primera causa de muerte en Colombia y presentan un comportamiento muy especial según la condición socioeconómica. ${ }^{21}$ En el anciano es más frecuente la disfunción diastólica además, de otras alteraciones del sistema circulatorio como lo son también las enfermedades coronarias, hipertensión arterial, enfermedades valvulares y arritmias. Así mismo, son comunes las enfermedades del sistema respiratorio, las entidades digestivas y renales siendo estas patologías un posible factor que modifique la planeación de un tratamiento. ${ }^{22-26}$

El sistema digestivo y el hepático inician una serie de cambios en deterioro que son producidos por los procesos normales de envejecimiento y acelerados por el consumo de alimentos de toda clase. ${ }^{27-29}$ Adicionalmente, es necesario hacer referencia a un fenómeno que actualmente es común en el adulto mayor como lo es la polifarmacia definida como el hábito o la necesidad de consumir dos o más fármacos al mismo tiempo debido a la presencia de varias entidades patológicas en un mismo cuerpo. Estas deficiencias producen como consecuencia Reacciones Adversas Medicamentosas (RAM), las cuales son efectos nócivos para la salud general y bucal del paciente, que de ante mano ya está vulnerada por su condicion de enfermo. ${ }^{29,30}$

Así mismo, es frecuente la alteración de la salud mental en la población del adulto mayor por desordenes mentales como depresión, demencia senil y esquizofrenia paranoide, entre otras. Uno de los motivos por los cuales la población del adulto mayor llega a este estado de total dependencia se da porque en su juventud carecieron de estímulos educativos o de aprendizaje que incentivaran a generar una constante actividad, por lo que no se generó el ejercicio cognitivo necesario para tener una buena salud mental. ${ }^{31,32}$

En relación con la cavidad bucal, los problemas que con mayor frecuencia afectan a los adultos mayores, además de los propios del envejecimiento, son la caries dental y la enfermedad periodontal, ${ }^{33,34}$ los cuales se observan hasta en el $88 \%$ de los adultos mayores que tienen dientes. ${ }^{35}$ Estas entidades pueden limitar la alimentación y repercutir negativamente en el estado nutricional y funcional; también bajan la autoestima por la apariencia personal y dificultan la comunicación al ser irreversibles e influir sobre la calidad de vida. ${ }^{35}$

La salud bucal no sólo se refiere al mantenimiento del mayor número de dientes naturales posibles en la boca, sino también al buen estado de los demás tejidos y órganos por lo que es necesario cumplir con rutinas de higiene que incluyen un cepillado y uso de seda dental adecuado, limpieza de prótesis en caso que las haya, uso de enjuagatorio y control odontológico. $^{23,36}$ Para la sociedad en general, los problemas de salud bucal originan un impacto social y económico desfavorable que generan dolor local, infección, halitosis, perdida de piezas dentarias y ocasionan problemas para masticar y hablar. ${ }^{37,38}$

El presente estudio pretendió establecer la influencia en la calidad de vida, de las necesidades de tratamiento bucal en los adultos mayores institucionalizados del municipio de Floridablanca, Santander (Colombia), a la luz de los recién denominados "Centros Vida" que pretenden mejorar la calidad de vida de este grupo poblacional. ${ }^{39-41}$

\section{MATERIALES Y MÉTODOS}

Se realizó un estudio observacional descriptivo de corte transversal en adultos mayores institucionalizados en seis hogares geriátricos "Centros Vida" ubicados en el municipio de Floridablanca, Santander (Colombia). ${ }^{42,43}$ La muestra estuvo conformada por todos los individuos que cumplieron con los criterios de inclusión, un total de 111 personas. Como criterio de inclusión se tuvo en cuenta a todos los adultos mayores institucionalizados en hogares geriátricos de Floridablanca que estuvieran internos o asistieran a la institución en el día. No se incluyeron los adultos mayores con enfermedad mental que les impidiera tomar decisiones o firmar el consentimiento informado y adultos mayores con limitación en la apertura bucal que dificultara la realización del examen clínico intraoral.

El instrumento diseñado tenía componentes similares a una historia clínica en el que anexaron algunos exámenes adicionales como flujo salival estimulado y no estimulado, estado de tejidos blandos según su propia descripción (normal o anormal), enfermedad sistémica presente, estado de prótesis dental según los criterios adaptada, mal estado, ausencia de soporte, estabilidad o retención, estado periodontal según el Índice Comunitario de Necesidad de Tratamiento Periodontal (CPTIN), higiene bucal al evaluar halitosis a un palmo, un metro o tres metros, higiene de lengua según cobertura, índice de placa bacteriana bueno, regular o malo y estado dental según la OMS. Además, se aplicó el cuestionario Geriatric Oral Health Assesment Index (GOHAI), que indaga acerca de la calidad de vida relacionada con la salud bucal de la persona examinada, según la auto percepción; se considero un GOHAI alto si el puntaje era mayor o igual a 57 y bajo si era menor que este.

Previa calibración de los investigadores, se efectuó la prueba piloto para estandarizar los procedimientos, verificar el instrumento y revisar la comprensión de 
las preguntas del GOHAI. Se recolectó la información relacionada con variables socio demográficas y una vez la persona aceptaba ser participe del estudio se realizaba el examen clínico bucal de forma sistemática; este examen se realizó en las instalaciones de la institución geriátrica.

La información se digitó en una base de datos en Excel creada para tal fin. Posteriormente, se exportó al programa estadístico SPSS 18 para su respectivo análisis. Para el análisis estadístico univariado se realizó una descripción de las características evaluadas mediante promedios y porcentajes según la naturaleza de las variables. En el bivariado se aplicó el Test de Chi Cuadrado o Test Exacto de Fisher con un valor de $\mathrm{p} \leq 0,05$ como estadísticamente significativo. ${ }^{44}$

\section{Consideraciones éticas}

Según la Resolución 08430 de octubre de 1993 emitida por el Ministerio de Salud en la cual se establecen las normas científicas, técnicas y administrativas para investigación en salud se consideró que esta fue una investigación de riesgo mínimo, ya que se recolectaron datos a través de procedimientos comunes, consistentes en examen clínico y se aplicó un cuestionario. A todos los participantes se les explicó el objetivo del trabajo y firmaron el consentimiento informado.

\section{RESULTADOS}

Según las variables sociodemográficas la población estuvo integrada por $62(55,9 \%)$ mujeres y 66 (59,5\%) personas procedían de la zona rural. El promedio de la edad fue 78,1 años, predominó el nivel primario con más de la mitad de las personas (Tabla 1).

Se encontró que $69(62,2 \%)$ personas necesitaban tratamiento especial o modificación del tratamiento por su enfermedad sistémica. A nivel general, 15 $(13,5 \%)$, requerían un manejo clínico de sus tejidos blandos; además, $42(37,8 \%)$ necesitban un tratamiento especial por flujo salival. De las $36(32,4 \%)$ personas que presentaban prótesis, necesitan reparación de ésta y $35(31,5 \%)$ requerían una prótesis total. En $12(44,4 \%)$ personas era necesario un raspaje y alisado radicular a campo cerrado y un manejo preventivo con educación en higiene bucal en 96 $(86,5 \%)$ individuos (Tabla 2$)$.

La calidad de vida de la población estudiada fue inferior a 57 y evidentemente, se vió alterada por frecuencias elevadas en las necesidades de tratamiento periodontal, estado de prótesis, manejo salival e intervención de tejidos blandos. Sin embargo, no se encontró una relación entre la calidad de vida relacionada con la salud bucal y la enfermedad sistémica (Tabla 3).
Tabla 1. Características socio demográficas de la población

\begin{tabular}{lc}
\hline \multicolumn{1}{c}{ Variables } & $\mathbf{n}(\%)$ \\
\hline Género & \\
Femenino & $62(55,9)$ \\
Masculino & $49(44,1)$ \\
Instituto & \\
1 & $5(4,5)$ \\
2 & $9(8,1)$ \\
3 & $12(10,8)$ \\
4 & $34(30,6)$ \\
5 & $40(36,0)$ \\
6 & $11(9,9)$ \\
Estrato & \\
2 & $99(90,0)$ \\
3 & $11(10,0)$ \\
Procedencia & \\
Rural & $66(59,5)$ \\
Urbana & $45(40,5)$ \\
Nivel educativo & \\
Primaria & $60(54,1)$ \\
Secundaria & $12(10,8)$ \\
Universitaria & -- \\
Ninguno & $39(35,1)$ \\
\hline
\end{tabular}

Tabla 2. Necesidades de tratamiento

\begin{tabular}{lc}
\hline \multicolumn{1}{c}{ Variables } & $\mathbf{n}(\%)$ \\
\hline $\begin{array}{l}\text { Necesidad de tratamiento por enfermedad } \\
\text { sistémica }\end{array}$ & $69(62,2)$ \\
Necesidad de intervencion de tejidos blandos & \\
y duros & $22(19,8)$ \\
Biopsia & $6(5,4)$ \\
Manejo clínico & $15(13,5)$ \\
Manejo quirúrgico & $8(7,2)$ \\
Necesidad de manejo salival & $42(37,8)$ \\
Necesidad de prótesis & \\
No requiere & $7(6,3)$ \\
Reparación & $36(32,4)$ \\
Prótesis parcial & $9(8,1)$ \\
Prótesis total & $35(31,5)$ \\
Necesidad de tratamiento periodontal & \\
Instrucción de higiene oral & $3(11,1)$ \\
Raspaje coronal, detartraje & $8(29,6)$ \\
Raspaje y alisado radicular, campo cerrado & $12(44,4)$ \\
Raspaje y alisado radicular complejo, campo & $4(14,8)$ \\
abierto & \\
Necesidad de manejo preventivo & $7(6,3)$ \\
No necesita & $25(22,5)$ \\
Limpieza y pulido & $57(51,4)$ \\
Control de placa & $96(86,5)$ \\
Educación en higiene oral & \\
\hline
\end{tabular}


Tabla 3. Frecuencias de autopercepción y calidad de vida relacionada con la salud bucal

\begin{tabular}{|c|c|c|c|}
\hline Necesidades de tratamiento & $\begin{array}{c}\text { No necesita atención } \\
\text { GOHAI } \geq 57 \\
\text { n (\%) }\end{array}$ & $\begin{array}{c}\text { Si necesita atención } \\
\text { GOHAI }<57 \\
\text { n }(\%)\end{array}$ & $\mathbf{P}^{\mathbf{a}}$ \\
\hline Total de participantes & $11(9,9)$ & $100(90,1)$ & \\
\hline \multicolumn{4}{|l|}{ Necesidad de manejo salival } \\
\hline No & $6(8,8)$ & $62(91,2)$ & \multirow[t]{2}{*}{0,82} \\
\hline $\mathrm{Si}$ & $5(11,9)$ & $37(88,1)$ & \\
\hline \multicolumn{4}{|c|}{ Necesidad de intervención de tejidos blandos } \\
\hline No & $9(10,1)$ & $80(89,9)$ & \multirow[t]{2}{*}{0,88} \\
\hline $\mathrm{Si}$ & $2(9,1)$ & $20(90,9)$ & \\
\hline \multicolumn{4}{|c|}{ Necesidad de manejo odontológico especial por presencia de enfermedad sistémica } \\
\hline No & $5(11,9)$ & $37(88,1)$ & \multirow[t]{2}{*}{0,58} \\
\hline $\mathrm{Si}$ & $6(8,8)$ & $63(91,2)$ & \\
\hline \multicolumn{4}{|l|}{ Necesidad de uso de prótesis } \\
\hline Prótesis completa & - - & $35(100)$ & \multirow{4}{*}{0,70} \\
\hline Prótesis parcial & $3(33,0)$ & $8(66,0)$ & \\
\hline Reparación de prótesis & $5(13,9)$ & $31(86,1)$ & \\
\hline No requiere prótesis & -- & $8(100)$ & \\
\hline \multicolumn{4}{|l|}{ Necesidad de tratamiento periodontal } \\
\hline Instrucción de higiene oral & - - & $3(12,5)$ & \multirow[t]{4}{*}{0,47} \\
\hline Raspaje coronal & $2(66,7)$ & $6(25)$ & \\
\hline Raspaje y alisado & $1(33,3)$ & $11(46,8)$ & \\
\hline Tratamiento complejo & - - & $4(16,7)$ & \\
\hline \multicolumn{4}{|l|}{ Diagnóstico de prótesis } \\
\hline Prótesis adaptada & $3(11,5)$ & $23(88,5)$ & \multirow[t]{5}{*}{0,70} \\
\hline Mal estado de prótesis & $1(3,3)$ & $29(96,7)$ & \\
\hline Ausencia de soporte & $16(88,9)$ & $2(11,1)$ & \\
\hline Ausencia de estabilidad & $3(11,1)$ & $24(88,9)$ & \\
\hline Ausencia de retención & $2(8,3)$ & $22(91,7)$ & \\
\hline
\end{tabular}

a: $\mathrm{Chi}^{2}$.

\section{DISCUSIÓN}

Si bien en este trabajo no se encontró una relación entre la calidad de vida relacionada con la salud bucal y la necesidad de tratamiento, si se observó que un alto porcentaje de pacientes requerían la modificación del tratamiento odontológico debido a la enfermedad sistémica que presentaban. Cardona y colaboradores han sugerido que la mayor parte de los adultos mayores padecen de hipertensión arterial, seguido por problemas articulares; mencionan que el 43,1\% de la población adulta mayor de Medellín sentía que su vida diaria había sido normal, además que las alteraciones de los sentidos no afectaban en nada sus actividades. ${ }^{45}$ Además, se ha evidenciado que el $39,9 \%$ las personas adultas mayores no presentan un control sobre su vida. ${ }^{46}$

Los resultados de esta investigación sugieren que la enfermedad sistémica alteraba la calidad de vida de las personas evaluadas. Wang y colaboradores, en 2009, establecieron el impacto de la hipertensión sobre la salud y calidad de vida, demostraron que cuando hay hipertensión se altera significativamen- te la salud mental y la función social del individuo. ${ }^{47}$

La salud bucal del adulto mayor se ve deteriorada por el paso de los años y por los efectos fisiológicos propios de la edad, a esto se le unen factores externos que pueden acelerar o retrasar este deterioro. Uno de esos factores, es llegar a una institución, lejos de la familia y del control que esta podría tener sobre él, como lo mencionan Cardona y colaboradores. ${ }^{45}$ Se ha establecido que en ocasiones, la falta de capacitación del personal encargado se considera como un factor coadyuvante para el deterioro de la salud bucal. ${ }^{45}$ Todos los adultos mayores analizados en esta investigación tuvieron algún tipo de necesidad, pero la más evidente fue la de enseñanza en higiene bucal, que si es bien manejada y con la adecuada capacitación, podría ser cubierta por los cuidadores involucrados en cada institución.

Strauss y Hunt evaluaron a 1000 adultos mayores en Estados Unidos y establecieron un orden de importancia según la afectación positiva o negativa por su buen o mal estado de salud bucal; cuando los encuestados presentaban buena salud bucal, 
consideraban que esta afecta de manera positiva en primer grado de importancia la apariencia, seguida por su alimentación, contrario al caso de no presentar una adecuada dentición era más importante la masticación y en última la apariencia. ${ }^{48}$

Se ha evidenciado una relación estrecha entre la calidad de vida y el estado de salud bucal en problemas dentales y gingivales relacionados con la percepción al aplicar un instrumento de calidad de vida relacionada con la salud oral (GOHAI). ${ }^{49,50}$ Así mismo, Smith y colaboradores encontraron una significancia estadística al relacionar la calidad de vida relacionada con la salud oral con la edad al aplicar el Oral Health Impact Profile (OHIP14), dado que al aumentar la edad se genera una perdida de dientes que hace precaria la función..$^{51}$ Zheng y colaboradores analizaron los factores asociados a la salud bucal con la calidad de vida en adultos mayores con dolor orofacial donde hubo una disminución de la calidad de vida en pacientes que reportaron dolor con una duración menor a un mes, con una severidad intensa. ${ }^{52}$

El presente estudio no encontró diferencias en la puntuación de GOHAI probablemente por la forma en que ésta fue categorizada o posiblemente, porque toda la población fue institucionalizada y en su mayoría de estratos socioeconómicos bajos. Aranzazu y colaboradores determinaron también una baja percepción de calidad de vida en adultos mayores no institucionalizados al tener que dejar o cambiar de actividades pero estos autores, no utilizaron el GOHAI. ${ }^{53}$

La caries dental fue uno de los principales motivos de pérdida dental, genera la necesidad de obtención de prótesis ya sea total o parcial removible en un $85 \%$, de los adultos mayores institucionalizados lo que coincide con los resultados encontrados por Williams y Montaño. ${ }^{54}$ Uno de los factores asociados a caries dental es el acúmulo de placa bacteriana que también se relaciona con la enfermedad periodontal. Todos los individuos con dientes que fueron evaluados presentaron acúmulos de placa bacteriana, resultados comparables con el trabajo realizado por Concha y Camargo en el que el $85,7 \%$ de la población evaluada registró algún marcador periodontal, ${ }^{3}$ así mismo ocurrió en el $96 \%$ de los adultos mayores evaluados en el Tercer Estudio Nacional de Salud Bucal (ENSAB III) y en otro estudio donde el $87,1 \%$ de los adultos mayores tenían necesidad de atención por enfermedad periodontal diagnosticada según el índice CPITN. ${ }^{55,56} \mathrm{Al}$ tener en cuenta que en su mayoría los dientes están ausentes, los pacientes tienen necesidades de manejo de prótesis igualmente, así mismo las necesidades de manejo periodontal son altas, teniendo en cuenta que solo 27 pacientes tenían dientes y el $100 \%$ de ellos registran alguna necesidad de manejo periodontal.

\section{Conclusiones}

En relación con las necesidades de tratamiento, la más alta prevalencia fue en la necesidad de educación en higiene bucal, seguido por tratamiento especial o modificación del tratamiento según su condición sistémica. Es importante mencionar una alta prevalencia en necesidad de tratamiento periodontal dentro de los pacientes dentados; por otra parte, dentro de la población desdentada la necesidad de reparación o uso de prótesis, seguido por tratamiento especial por flujo salival en última estancia. Por lo tanto, es de gran importancia la promoción de la salud y la prevención de la enfermedad en este grupo poblacional para mejorar su bienestar en relación con las necesidades de tratamiento bucal que se requieran.

\section{Agradecimientos}

Agradecemos a la Universidad Santo Tomás en Bucaramanga y al excelente equipo de trabajo del Semillero de Investigación SIFOUSTA que con su dedicación hicieron de esta investigación una inolvidable experiencia en donde el aprendizaje y el contacto humano fue constante.

\section{BIBLIOGRAFÍA}

1. Cámara de comercio de Bogotá. Colombia siglo XXI. 1991.

2. Organización Panamericana de la Salud, 1985.

3. Concha SC, Camargo DM. Análisis de la asociación entre la calidad de vida y condición oral de las personas mayores vinculadas a tres instituciones geriátricas de Bucaramanga: II Fase. Ustasalud 2007; 6: 75 - 86.

4. Organización de las Naciones Unidas. Comisión para el Desarrollo Social. Estrategia Internacional para la Acción 2002 para el Envejecimiento; 2003: p. 1 - 3.

5. Organización Mundial de la Salud. Departamento de Promoción de la Salud. Salud y Envejecimiento. Un documento para el debate. Segunda Asamblea Mundial. España. 2002 [fecha de acceso: marzo de 2011]. URL Disponible en: http://www.redadultosmayores.com.ar-buscadorfiles-SA-LUD093-Edwards.pdf

6. Cardona D, Estrada A. Calidad de vida y condiciones de salud de la población adulta mayor de Medellín. Biomédica 2006; 26: 206 - 215.

7. Nieto M, Abad M, Esteban A, Tejerina M. Psicología para Ciencias de la Salud: estudio del comportamiento humano ante la enfermedad. Madrid: McGraw-Hill Interamericana; 2004.

8. Esquivel R, Ferez J, González A, Gazano A. Efecto de la depresión en la percepción de salud bucal y calidad de vida en adultos mayores. Revista ADM 2006; LXIII: 62 - 68.

9. Gómez M, Sabeh E. Calidad de vida. Evolución del concepto y su influencia en la investigación y la práctica. [fecha de acceso: marzo de 2011]. URL disponible en: http://inico.usal.es/publicaciones/pdf/calidad.pdf. 
10. Velarde-Jurado E, Avila-Figueroa C. Consideraciones metodológicas para evaluar la calidad de vida. Salud Pública de México 2002; 44: 448 - 463.

11. Guzmán L, Zarate O. Series en medicina bucal X. Odontología de calidad para los pacientes ancianos. Revista ADM 2005; LXII: 36 - 39.

12. Meller C. Importancia de la odontología preventiva en el adulto mayor: Una aproximación personal. Odontol Prev 2008; 1 : 73 - 82.

13. García H, Valdés S, García E, Fernández S, Fernández M. Problemas de salud pulmonar en adultos mayores. Hogar de Santovenia. Geroinfo. RNPS 2110 [fecha de acceso: marzo de 2011]; 4: 1 - 9. URL Disponible en: http://www. sld.cu/galerias/pdf/sitios/gericuba/salud_pulmonar_en_ ancianos.pdf

14. Villasana L, Flores R, Facio D. Programa de Atención al Adulto Mayor. El fenómeno del envejecimiento de la población en el estado de Nuevo León. Un reto de la política social 2006 [fecha de acceso: mayo de 2011]. URL disponible en http://www.nl.gob.mx/pics/pages/cuadernos_cds_ base/libro_programaadultomayor.pdf

15. Borges S, Gómez H. Uso de los servicios de salud por la población de 60 años y más en México. Salud Pública de México 1998; 40: 13 - 23.

16. Rockville MD. Department of health and human service. US. Public health service. National institute of dental and craniofacial research. National Institute of Health 2000; 5: 6.

17. Esquivel RI, Jiménez J. Necesidades de atención odontológica en adultos mayores mediante la aplicación del GOHAI. Revista ADM 2010; LXVII: 127 - 132.

18. Fuente-Hernández J, Sumano-Moreno O, Sifuentes-Valenzuela MC, Zelocuatecalt-Aguilar A. Impacto de la salud bucal en la calidad de vida de adultos mayores demandantes de atención dental. Universitas Odontológica 2010; 29: 83 - 92

19. Leitón Z, Ordoñez Y. Autocuidado de la salud para el adulto mayor, manual de información para profesionales. Centro de documentación OPS - OMS Perú 2003: 67 - 70.

20. Ghezzi E, Ship J. Systemic diseases and their treatments in the Elderly: Impact on oral health. J Pub Health Dent 2000; 60: 289 - 296

21. Jaramillo NI, Torres Y. Aspectos epidemiológicos de la enfermedad cardiovascular: factores de riesgo a la luz de Framingham. CES Medicina 2004; 18: 45 - 68.

22. Jaramillo CJ. Enfermedades Cardiovasculares en el Anciano [fecha de acceso: mayo de 2011]. URL Disponible en: http://www.laboratoriosamerica.com.co/medicosenlinea/congreso2003/descargas/EnfermedadesCardiovascularesAnciano.doc

23. Pavés V, Quintana M. Manejo odontológico de pacientes ancianos con riesgo sistémico. Revista Dental de Chile 2003; 94: 22 - 25.

24. Ministerio de Salud. Presidencia de la Nación. Enfermedades Infecciosas. Infección respiratoria aguda del adulto. Diagnóstico de infección respiratoria aguda del adulto. Guía para el equipo de salud [fecha de acceso: mayo de 2011] URL Disponible en: http://www.msal.gov.ar/htm nuevo/site/pdf/guia-ira-int.pdf.

25. Figueroa M, Varela L. Depresión en el adulto mayor. Estudio de una serie de pacientes de consultorio externo de medicina en el Hospital Nacional Cayetano Heredia. Revista Med Hered 1996: 7: $172-177$.

26. Rebollo P, Bobes JM. González P, Saiz P, Ortega F. Factores asociados a la calidad de vida relacionada con la salud (CVRS) de los pacientes en terapia renal sustitutiva (TRS). Nefrología 2000; XX: $171-181$.

27. Quintana A, Sánchez T, Quintana J, Reyes E, de la Guardia E. El adulto mayor en cirugía general. Rev Cubana Cir 2001; 40: $305-311$

28. Arias E, Gomez A, Jimenez C. Patologia Hepatobiliar. En: Sociedad Española de Geriatría y Gerontología. Tratado de Geriatría para Residentes. Madrid: International Marketing \& Communications; 2006. p. 581 - 588.

29. Berenguer M. La salud bucodental en la tercera edad. MEDISAN 1999; 3: 53 - 56.

30. Guitron M, Carrillo M. Reacciones adversas a medicamentos en la boca del adulto mayor. Revista ADM 2010; LXVII: $163-170$

31. Cisneros JM, Cobo J, Pujol M, Rodríguez J, Salavert M. Guía para el diagnóstico y tratamiento del paciente con bacteriemia. Guías de la Sociedad Española de Enfermedades Infecciosas y Microbiología Clínica (SEIMC) [fecha de acceso: junio de 2011]. URL Disponible en: http://apps.elsevier.es/watermark/ctl servlet? $\mathrm{f}=10$ \&pident articulo $=13098572$ \&pident usuario $=0$ \&pcontactid $=$ \&pident_revista $=28 \&$ ty $=143 \&$ accion $=$ L\&origen $=$ elsevier\&web $=$ www.elsevier.es\&lan $=$ es\&fichero $=28 \mathrm{v} 25 \mathrm{n} 02 \mathrm{a} 13098572 \mathrm{pdf001}$.pdf

32. Beck J, Kohout F, Hunt R. Identification of high caries risk adults: attitudes, social factors and disease. Int Dent J 1998; 38: 231 - 238.

33. Splieth C, Meller C. Importancia de medidas preventivas en el adulto mayor. Revista Dental de Chile 2003; 94: 8 $-12$.

34. Gómez R, Lara R. La transición demográfica en México y las especialidades odontológicas. Practica Odontol 1999; 20: $36-38$.

35. Marín D. Influencia de la boca en calidad de vida de los ancianos. Revista Asociación Colombiana de Gerontología y Geriatra 2002; 16: 148 - 152.

36. Cepeda LA, Garza R, Vega D. Programa de Atención al Adulto Mayor. Fenómeno del envejecimiento de la población en el estado de Nuevo León. Un reto en la política social. Monterrey: 2006 [fecha de acceso: marzo de 2012]. URL disponible en: http://www.nl.gob.mx/pics/pages/ cuadernos_cds_base/libro_programaadultomayor.pdf

37. García C, Maguiña E. Salud bucal del adulto. Revisión para profesionales del equipo de atención primaria. MPA e-Journal de Medicina Familiar y Atención Primaria Internacional 2008 [fecha de acceso: julio de 2011] ; 231 - 37. URL disponible en: www.idefiperu.org/mpa.html

38. Richie C, Burgio K, Locher J, Cornwell A, Thomas D, Hardin $\mathrm{M}$, et al. Nutritional status or urban homebound older adults. Am J Clin Nutr 1997; 66: 815 - 818.

39. Alcaldía de Floridablanca. Ubicación y límites de Floridablanca [fecha de acceso: agosto de 2011]. URL Disponible: http://www.floridablanca.gov.co/site/index. php?option $=$ com content\&view $=$ category\&layout $=$ bl og\&id $=132$ \&Itemi $\overline{\mathrm{d}}=222$

40. Alcaldía de Floridablanca. Demografia del Municipio de Floridablanca. [fecha de acceso: agosto de 2011]. URL Disponible en: http://www.floridablanca.gov.co/site/index.php?option $=$ com content\&view $=$ category\&layout $=$ blog\&id $=134 \&$ Itemi $\bar{d}=224$

41. Ley 1276 de 2009. Diario oficial No. 47223 de enero de 2009. Congreso de la República, Ley 687 del 15 de agosto de 2001. Criterios de atención integral del adulto mayor 
en los Centros Vida [fecha de acceso: agosto de 2011] URL disponible en: http://www.elabedul.net/Documentos/Leyes/2009/Ley_1276.pdf

42. Hosmer D, Lemeshow S. Applied Logistic Regression. New York: John Wiley \& Sons; 2000.

43. Kleinbaum DG, Kupper L, Morgenstern H. Epidemiologic Research: Principles and Quantitative Methods. New York: John Wiley \& Sons; 1982. p. 52 - 93.

44. Pagano M, Gauvreau K. Principles of Bioestatistics. Belmont: Duxbury Press; 1995. p. 35 - 44, 257 - 263.

45. Cardona D, Estrada A, Chavarriaga LM, Ordoñez JE, Osorio JJ, Segura AM. Situación de salud del adulto mayor institucionalizado Medellín 2008. Medellin: Editorial CES; 2010.

46. Giménez J. Esquivel R. Martínez N. Percepción de salud bucal, calidad de vida y bienestar subjetivo en ancianos. Revista ADM 2006; LXIII: 181 - 184.

47. Wang R, Zhao Y, He X, Ma X, Yan X, Sun Y, et al. Impact of hypertension on health-related quality of life in a population-based study in Shanghai, China. Public Health 2009; 123: 534 - 539.

48. Strauss $P$, Hunt R. Understanding the value of teeth to older adults: Influences on the quality of life. J Am Dent Assoc 1993; 124: 105 - 110.

49. Piuvezam G, De Lima KC. Self-perceived oral health status in institutionalized elderly in Brazil. Arch Gerontol Geriatr 2012; 55: 5 - 11.

50. Atchinson K, Dolan T. Development on the geriatric oral health assessment index. J Dent Educ 1990; 54: 680 - 686.

51. Smith B, Baysan A, Fenlon M. Association between oral health impact profile and general health scores for patients seeking dental implants. J Dent 2009; 37: 357 - 359.

52. Zheng J, Wong CM, Lam LK. Key factors associated with oral health-related quality of life (OHRQOL) in Hong Kong Chinese adults with orofacial pain. J Dent 2011; 39: 564 $-671$.

53. Aranzazu GC, Martínez CA, Uribe MM, Boada H. Percepciones de la salud general, oral y visual del adulto mayor que asiste a las clínicas de la universidad Santo Tomás, factores que influencian su calidad de vida. Ustasalud 2007; 6: 88 - 97 .

54. Williams E. Montaño M. Caries en el adulto mayor, un reto para la odontología. Revista Mexicana de Odontología Clínica 2007; 12: 10 - 15.

55. Ministerio de Salud, Tercer Estudio Nacional de Salud Bucal ENSAB III; 1998.

56. Arlette S. Zunzunegui M. Detección de necesidades de atención bucodental en ancianos mediante la autopercepción de la salud oral. Revista Multidisciplinar de Gerontología 1999; 9: 216 - 224.

Correos electrónicos de los autores:

Gloria Cristina Aránzazu Moya: aranzazugloria@yahoo.es Anne Alejandra Hernández Castañeda: anne.usta@hotmail.com Maira Alejandra Gutiérrez Sánchez: maleja.gut@hotmail.com Daniel Felipe Agudelo Prada: danex30@hotmail.com
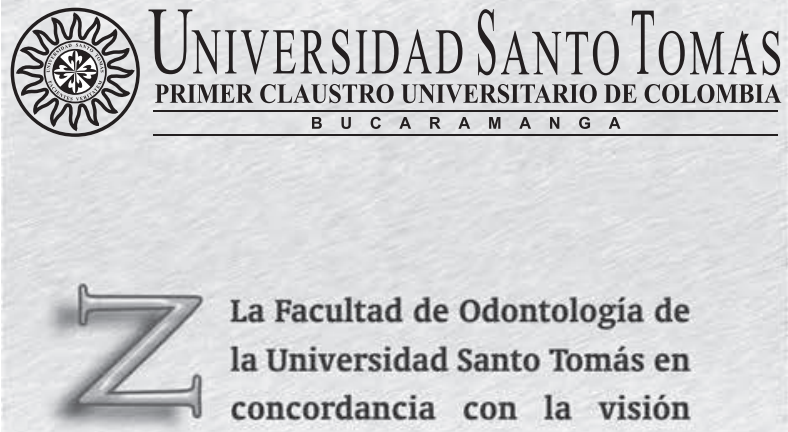

La Facultad de Odontología de la Universidad Santo Tomás en concordancia con la visión institucional aspira lograr:

Un compromiso efectivo de la comprensión renovada del hombre colombiano, su mundo y sus valores, en un contexto de salud integral para todos; el reconocimiento nacional e internacional, como comunidad académica, a través de la investigación, la producción intelectual y el intercambio académico; liderazgo en los procesos relacionados con la salud bucal de la comunidad, a nivel regional y nacional; la cualificación permanente de sus servicios a la comunidad para responder efectiva y oportunamente a sus usuarios; el fortalecimiento permanente de su accionar pedagógico mediante el desarrollo humano integral, la aplicación de tecnologias de punta y la proyección comunitaria. 